\title{
COMMISSION 4: EPHEMERIDES(EPHÉMÉRIDES)
}

President: H. Kinoshita

Vice President: M. Standish

Organizing Committee: V.K.Abalakin, T. Fukoshima, G.A. Krasinsky, P.K. Seidelmann, J.-E. Arlot, He Miao-Fu, J.H. Leiske, B.D. Yallop, J. Chapront, G.H. Kaplan, H. Schwan

\section{Introduction}

During 1994-1996 Ephemerides have been intensively developed for all celestial bodies in the solar system in both analytical and numerical ways, except an analytical ephemeris of Pluto. Because of the shortage of allotted pages this report covers only the reviews of planetary ephemerides. Ephemerides of natural satellites and other bodies will be included in the next report as six years' achievements. Conventional reports from institutions and individual members were published in the Newsletter of Commission 4.

In preparing this report the reviews by $M$. Standish and P. Bretagnon are highly appreciated.

\section{Analytical Ephemerides of planets (Bretagnon)}

Several studies have been developed in the construction of classical and general planetary theories during these last three years and these theories have been used in the calculation of the precession-nutation quantities and in the determination of the relations between the barycentric and geocentric reference frames.

\subsection{CLASSICAL PLANETARY THEORY}

\subsubsection{Classical planetary theory with only one argument}

A planetary theory of the planets Jupiter, Saturn, Uranus and Neptune has been built by Simon (1996). It is a classical planetary theory where the perturbations are computed in the form of Poisson series of only one angular variable, using the iterative method of Simon and Joutel (1988). This method allows a best convergence of the mutual perturbations Jupiter-Saturn.

Perturbations by the inner planets developed up to the third order of the masses are issued from VSOP82 (Bretagnon, 1982). They are introduced in the iterations. So, are computed the perturbations by the inner planets to the order 4 of the masses and a part of the perturbations of superior orders.

The theory is built with modern values of the planetary masses and fitted to the numerical integration DE245 of the Jet Propulsion Laboratory (Standish, 1994).

For Jupiter, Uranus and Neptune the precision is better than $0^{\prime \prime} .01$ over the time span $+1000,+3000$ and better than 0.8 over $-4000,+8000$. For Saturn, the precision is about $0 \prime .02$ over $+1000,+3000$ and about $2^{\prime \prime}$ over $-4000,+8000$. The gain in precision, in connection with the best analytical theories actually available, as VSOP87 (Bretagnon and Francou, 1987) and JASON84 (Simon and Bretagnon, 1984), is about 10 .

\subsubsection{Classical planetary theory using elliptic functions}

A new method (Chapront and Simon, 1996) to represent the series of the planetary motions has been developed by using elliptic functions. For the pair Jupiter-Saturn, this method notably improves the convergence of the classical series of the celestial mechanics. The case of a pair of planets with a high ratio of the semimajor axes (Venus-Earth) has been studied. With respect to the classical developments in function of the mean longitudes, the series are much less bulky by substituting an "elliptic argument" for the "time". 


\subsection{GENERAL PLANETARY THEORY}

\subsubsection{General planetary theory and rotation of the Earth}

At present, the analytical solutions of the lunar motion are built by using classical theories of the motion of the Sun and of the planets valid over only a few thousands of years. Therefore, there exists no precise solution of the lunar motion over some million years and it is not possible to build the precessionnutation series over such time spans. Brumberg and Ivanova (1996) propose a method of construction of a general planetary theory which would enable one to build together the quasi-periodic series describing the translatory motion of the major planets and the Moon and the series describing the rotation of the Earth. Thus this approach gives the evolution of the planetary and lunar orbits and the theory of the Earth's rotation presented by means of the series in powers of the evolutionary variables with quasi-periodic coefficients.

\subsubsection{General planetary theory using elliptic functions}

Klioner (1996) has built a general planetary theory with the aid of the elliptic functions and integrals. He compares his results with the purely trigonometric form solutions. The expansions of elliptic function give certain level of compactification of the resulting series in the case of mutual perturbation of a pair of planets. However, the level of "compactification" is not very high $(<2$ for actual major planets) and the elliptic function does not give any compactification in case of mutual perturbations of a triplet of planets.

\subsection{METHODS OF APPROXIMATION OF THE PLANETARY SOLUTIONS}

Chapront (1995) has built a Fourier-Chebychev approximation method and applied it to the motion of the five outer planets. From the results of the JPL numerical integration DE200, a frequency analysis is performed over a time span of a hundred or so periods of the concerned body. The mixed solutions - Fourier and Poisson series - represent the planetary motion over several centuries with an accuracy better than $0^{\prime \prime} .002$ by series comprising less than 150 terms.

\subsection{ROTATION OF THE RIGID EARTH}

For the last three years, nutation series for a rigid Earth have been computed by different methods from the analytical planetary theories (VSOP82 or VSOP87) and the analytical lunar theory (ELP). Souchay and Kinoshita (1996a, b) used Hamiltonian methods. Williams (1994, 1995), Roosbeek and Dehant (1996), Bretagnon, Rocher and Simon (1996) compute torques on the oblate Earth. It must also be mentioned that Hartmann and Soffel (1994) proceed by numerical integration from the tidal potential. These various solutions are very close one another, the differences does not exceed 1 milliarcsecond.

\section{Numerical Ephemerides of Planets (Standish)}

\subsection{CAL TECH / JET PROPULSION LABORATORY; PASADENA, CALIFORNIA}

JPL's latest planetary and lunar ephemeris, DE403, (Standish et al.,1995) was integrated from 3000 BC to $3000 \mathrm{AD}$ and has been made available via anonymous ftp [navigator.jpl.nasa.gov - 128.149.23.82] and in limited quantity on CDrom.

The ephemeris was fit to nearly 40,000 observations, including optical transits, astrolabe, astrometry, occultation timings, radio astrometry, radar ranging, spacecraft, frame-tie measurements, and lunar laser ranging. DE403 is referenced directly onto the International Celestial Reference Frame (ICRF) by the inclusion of VLBI measurements of spacecraft in orbit around Venus (Magellan) and Mars (Phobos) and by the inclusion into the fits of the frame-tie determination Folkner et al. (1995).

The ephemeris is still integrated on the Univac 1108, because that computer has a 60-bit mantissa in double precision. We find that modern workstations with only 53-bit mantissas do not have the precision necessary for integrating the motion of the moon at the sub-centimeter level, necessary for reducing and adjusting to modern Lunar Laser Ranging data. Quadruple precision is about 100 times slower - too slow when integrating many centuries of motion. A new formulation by Fukushima (1996, "Generalization of Enck's method and its application to the orbital and rotational motions of celestial bodies"), gives an 
error of only a few centimeters after 25 years, as opposed to a few meters when using cartesian coordinates. Further alternatives are being explored.

\subsection{HARVARD / SMITHSONIAN CENTER FOR ASTROPHYSICS; CAMBRIDGE, MASSACHUSETTS}

In a paper submitted to AJ in May, we have a table of the rotations and rotation rates between pairs of ephemerides as determined by a direct comparison of tabulated Earth-Sun vectors over 12 years. The paper is about pulsar positions, so the emphasis is on ephemerides used for timing analysis: PEP311, PEP740, PEP740R, DE96, DE118, and DE200. The results were obtained two ways: first by a global solution with the offset and rate for the whole span, and second by making 12 yearly fits for just the offsets and pulling a mean slope out. They agree. There is some systematic waviness apparent in the second approach, but the size of the residuals after taking out just the linear drift is at the level of $10^{* *}-10$ rad for a typical pair of ephemerides. For PEP740 and DE200, even though they are each well over 10 years old, the rotation rates between the two are only $0.000,0.002$, and -0.005 arcseconds per century for the $x_{-}^{-}, y_{-}$, and $z_{-}$axes, respectively, in the sense that the mean motion of the earth is slightly less for PEP740.

\subsection{UNITED STATES NAVAL OBSERVATORY}

Currently the U.S. Naval Observatory is working towards the improvement of planetary ephemerides by providing reliable masses for the asteroids. This year we have produced the first reliable mass for 15 Eunomia, and are working to provide improvements to the masses of 1 Ceres, 2 Pallas, and 4 Vesta. The encounters that might provide the masses of several other large asteroids such as 10 Hygiea and 16 Psyche are currently being evaluated for their usefulness. As a part of this work new ephemerides are being produced for 15 of the largest asteroids. In addition, the U.S. Naval Observatory has begun an effort to write a modern ephemerides program based on the Planetary Ephemerides Program.

\subsection{INSTITUTE OF APPLIED ASTRONOMY; ST. PETERSBURG, RUSSIA}

A Numerical theory of the Moon and planets has been constructed by a numerical integration which also includes equations for the rotational motion of the Moon and the Earth. The influence of tesseral harmonics of the geopotential upon the lunar orbit is taken into account; these harmonics generate semidiurnal perturbations of the order $2 \mathrm{~cm}$ and an additional secular trend in lunar longitude about 0.8 $\operatorname{arcs} / \mathrm{cy}$.

The reference frame of the theory was made to coincide with that of DE200. In this reference frame the initial coordinates and velocities of the inner planets were improved from an analysis of available ranging data (including data from Mariner 9 spacecraft and Martian landers Viking 1 and 2). From these data it appears possible to derive corrections to the mass of Jupiter and to the four elements of Jupiter (excepting the inclination and node). The results are presented by E. Pitjeva at IAU colloquium 165 (July 1-5, 1996) in the paper "The Ephemerides of the Inner Planets from Spacecraft Range Data and Radar Observations 1961-1965".

The parameters of lunar orbital and rotational models were improved from an analysis of LLR data. A reference lunar ephemeris was obtained which presents LLR data (1969-1994 yy) with an rms error of about 2 nsec. A preliminary estimation of the harmonics of the lunar gravitational potential as well as the main nutation coefficients of the Moon was carried out (Aleshkina et al., 1996).

\section{References}

Aleshkina E.Yu.,Krasinsky G.A., and Vasilyev M.V. : 1996, in IAU Colloquium No. 165, Poznan.

Brumberg, V. A., and Ivanova, T. V. : 1996, in IAU Colloquium No. 165, Poznan.

Chapront, J. : 1995, A\&A Suppl. Series, 109, 181.

Chapront, J., Simon, J.-L. : 1996, Celest. Mech, 63, 171.

Folkner,W.M., Charlot,P., Finger,M.H., Williams, J.G., Sovers,O.J., Newhall, XX, and Standish,E.M. : 1993, A\&A, 287, 279.

Fukushima,T. : 1996, in Proceedings of the 28th Symposium on Celestial Mechanics, Tokyo, Japan Kinoshita and Nakai (eds.), 64

Hartmann, T., Soffel, M. : 1994, A. J., 108(3), 1115

Klioner, S. A. : 1996. Celest. Mech, (in press).

Moisson, X. : 1996, in IAU Colloquium No. 165, Poznan. 
Roosbeek, F., Dehant, V. : 1996, Celest. Mech, (in preparation).

Souchay, J., and Kinoshita, H. : 1996a, $A \& A$ (in press).

Souchay, J., and Kinoshita, H. : $1996 \mathrm{~b}, A \& A$ (in press).

Simon, J.-L., Joutel, F., and Bretagnon, P. : 1992, A\&A, 265, 308.

Simon, J.-L. : 1996, in , Dynamics, Ephemerides and Astronomy of the Solar System S. Ferraz-Mello et al. (eds), 49.

Standish,E.M., Newhall, X X, Williams,J.G. and Folkner,W.M. : 1995, JPL IOM,314,10.

Williams, J. G. : 1994, A. J., 108(2), 711.

Williams, J. G. : 1995, A. J., $110(3), 1420$. 